\title{
A Comparison of Covert and Overt Attention as a Control Option in a Steady- State Visual Evoked Potential-based Brain Computer Interface
}

\author{
S. P. Kelly ${ }^{1}$, E. Lalor ${ }^{1,2}$, C. Finucane ${ }^{3}$, R. B. Reilly ${ }^{1}$ \\ ${ }^{1}$ Department of Electronic \& Electrical Engineering, University College Dublin, Ireland. \\ ${ }^{2}$ Media Lab Europe, Dublin, Ireland. \\ ${ }^{3}$ Medical Physics \& Bioengineering Dept., St. James’ Hospital, Dublin, Ireland.
}

\begin{abstract}
EEG data were recorded from occipital scalp regions of subjects who attended to an alternating checkerboard stimulus in one visual field while ignoring a similar stimulus of a different frequency in the opposite visual field. Classification of left/right spatial attention is attempted by extracting Steady-State Visual Evoked Potentials (SSVEPs) elicited by the stimuli to assess the potential use of such a spatial selective attention paradigm in a Brain Computer Interface (BCI). Experimental setup and analysis procedure in a previous study in which eye movement is permitted are replicated in order to quantify differences in classification performance using overt and covert attention. Four variations of the basic paradigm, involving both feedback and addition of extra mental load, are studied for comparison. The average accuracy is found to be reduced by $\sim 20 \%$ in the switch from overt to covert attention when no other specifications of the task are changed.
\end{abstract}

Keywords-Brain Computer Interface, EEG, Spatial Selective Attention, Steady-State Visual Evoked Potential

\section{INTRODUCTION}

For some people with very severe disabilities (e.g. amyotrophic lateral sclerosis or brainstem stroke), a brain computer interface (BCI) may be the only feasible channel for communicating with others and for environment control [1]. The most favorable brain imaging method employed in BCIs is electroencephalography (EEG), in which electrical signals of high temporal resolution are recorded noninvasively from the scalp. EEG-based BCIs generally utilize either reliably generated electrical responses such as eventrelated potentials (ERPs) and variations in cortical rhythms $[2,3,4]$, or self-regulatory activity such as slow cortical potentials [5]. The former design, being reliant on natural involuntary responses has the advantage of requiring no training, whereas the latter design normally demonstrates effectiveness only after periods of biofeedback training, wherein the subject learns to regulate the relevant activity in a controlled way.

One BCI solution, which has had considerable success in optimizing performance in terms of both speed and accuracy, relies on an involuntary response known as the Steady-State Visual Evoked Potential (SSVEP). This is a periodic response elicited by the repetitive presentation of a visual stimulus, at a rate of $6-8 \mathrm{~Hz}$ or more [6]. Though the existing SSVEP-based BCIs are exceptionally robust and convenient to implement [7], most designs require reliable control of eye movement - the subject makes selections by shifting gaze direction. This unfortunately rules out applicability to those whose severe disabilities extend to impaired ocular motor control. Furthermore, sophisticated gaze-tracking apparatus now available renders this type of "dependent BCI" redundant to some degree.

Though the specific underlying mechanisms of the SSVEP are as yet not well understood, there have been several accounts of its reactivity to cognitive variables such as attention, stimulus classification and memory search $[8,9]$. Of particular interest are reports of the SSVEP behaving as an index of visual-spatial selective attention. In [10], two sequences of alphanumeric characters were presented, superimposed on flickering backgrounds of different frequencies. It was found that when the subjects attended to the sequence in one visual field the amplitude of the SSVEP resulting from the flickering background on that side was enhanced by about a factor of two, compared to when the subject attended to the opposite side.

Visual selective attention is a psychophysical construct pertaining to the brain's ability to identify and focus on certain components of visual input to be processed preferentially at a given time. This is necessitated by the large amount of information transferred from the retina to higher processing areas of the brain (about $10^{8}-10^{9}$ bits per second) which, given the limited computational resources, is far too much to be processed in its entirety [11]. In particular, spatial selective attention may be understood in terms of a spotlight metaphor. Helmholtz [12] pointed out that the spotlight may move around a visual scene independent of gaze direction, i.e. components in peripheral vision may be selected for processing just as those in foveal vision. The term covert attention is used to describe attentional selection of regions of visual space outside the central foveal region.

In a previous study by the authors [13], the possibilities for one-dimensional control (i.e. binary decsions) were investigated in a task involving eye movement towards either of two checkerboard stimuli reversing at frequencies $17 \mathrm{~Hz}$ and $20 \mathrm{~Hz}$. SSVEPs were extracted from data acquired during several 25 -second trials in which subjects overtly attended to the left or right stimulus, and subsequently used in left/right classification. Accuracies based on a Fourier method for SSVEP extraction ranged from $54.2 \%$ to $92.2 \%$, with an average accuracy of $74.4 \%$ across six subjects. 
An interesting question arising from the abovementioned results of recent neuroscience research $[8,10]$ is whether covert attention, which is known to modulate SSVEP amplitude, can be used in a task such as that detailed in [13]. Due to the organization of the visual cortex, the spatial distribution and amplitude of electrophysiological responses to covertly attended visual stimuli differ from responses to stimuli in foveal vision. Thus adjustments to the paradigm are likely to be necessary in order to achieve optimum performance. It is nevertheless useful as an intuitive step towards covert attention-control in a BCI to quantify how much of a reduction in left/right classification performance would result from disallowing eye-movement, without changing the basic specifications of the task in [13].

In this paper the authors investigate the possibilities of one-dimensional control using covert attention in a paradigm for which effective control is achievable using overt attention.

\section{METHODOLOGY}

\section{A. Subjects}

15 subjects aged between 21 and 38 participated in the study. All had normal or corrected-to-normal vision.

\section{B. Experimental set-up}

Subjects were seated $180 \mathrm{~cm}$ from a large screen onto which was projected two $4 \times 4$ checkerboard patterns, as in Fig. 1. The left patterns reversed at a rate of $17 \mathrm{~Hz}$ and the right at $20 \mathrm{~Hz}$. The patterns were situated $8.2^{\circ}$ bilateral to a central fixation cross (cross to medial edge), centered on the horizontal meridian, and subtended a visual angle of $9.9^{\circ}$ vertically and $11.2^{\circ}$ horizontally. EEG signals were recorded from the $\mathrm{O} 1$ and $\mathrm{O} 2$ electrode positions using an electrode cap. These positions are situated over the left and right hemisphere of the primary visual cortex respectively [14]. Each channel, referenced to the right ear lobe, was amplified $(50 \mathrm{~K}), 50 \mathrm{~Hz}$ line filtered and band-pass filtered over the range $0.01-100 \mathrm{~Hz}$ by Biopac biopotential amplifiers [15]. The signals were then digitized at a rate of $256 \mathrm{~Hz}$ using a National Instruments DAQ system [16].

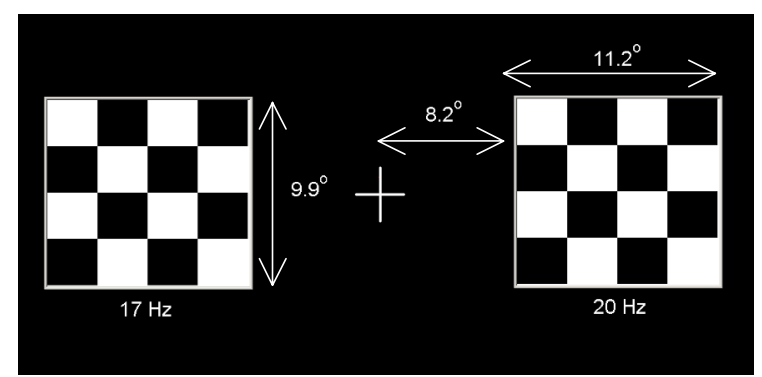

Fig. 1. Stimulus presentation.
Horizontal eye movements were monitored using horizontal Electrooculogram (EOG) recorded from two electrodes placed at the outer canthi of the eyes referenced to each other.

\section{Procedure}

Each subject underwent a total of 32 trials each of 25 seconds duration. For all trials the subject was required to covertly attend to the left or right checkerboard and to sustain this spatial attention for the entire 25 seconds. There were four variations of this basic task:

1) Standard: Left and right checkerboard patterns differed in no way except in frequency. No additional task was required of the subject.

2) Yellows: Embedded in the normal sequence of black and white phase reversals, a yellow and black stimulus appeared at random for one cycle of the stimulus. This occurred at intervals greater than one second to avoid confusion caused by a rapid succession of yellow targets. The subject was required to count the number of yellow target stimuli presented in the 25 -second period of each trial, and report the total at the end of the trial. Subjects were informed after each trial whether they counted correctly.

3) Feedback: Auditory feedback was provided to the subject using headphones. A double-chirp sound, the playspeed of which was proportional to the one-dimensional spectral ratio feature F1 explained in the following section, was repeatedly presented. Thus when the subject overtly attended the left stimulus the chirp slowed down and decreased in pitch, and the opposite occurred with overt attention to the right stimulus. Subjects were instructed to use the auditory feedback to aid them in covertly attending to the left or right standard checkerboard stimulus.

4) Feedback and yellows: Subjects were instructed to count yellow target stimuli while provided with auditory feedback.

All subjects began with the standard task type, but thereafter the order of task type was counterbalanced across subjects. For each task type subjects underwent 4 left trials and 4 right trials ordered alternately.

\section{Feature Extraction}

Each 25-second trial was divided into approximately 50 2 -second segments with 0.5 seconds overlap, each of which counts as a single case for which the feature is derived. Artifact rejection was carried out on the basis of the concurrently recorded EOG signal - segments containing an EOG deflection exceeding that which corresponds to lateral eye movements of about $5^{\circ}$ were rejected

The Fast Fourier Transform (FFT) for each segment was calculated and squared:

$$
X_{n}^{O 1}=\left|F F T\left(x_{n}^{O 1}(t)\right)\right|^{2} ; X_{n}^{O 2}=\left|F F T\left(x_{n}^{O 2}(t)\right)\right|^{2} .
$$


For comparison with previous overt attention results, the procedure was replicated as follows: A one-dimensional feature was extracted for each segment:

$$
F 1(n)=\log \left(\frac{X^{O 1}{ }_{n}(f 1)+X^{O 2}{ }_{n}(f 1)}{X^{O 1}{ }_{n}(f 2)+X^{O 2}{ }_{n}(f 2)}\right),
$$

where $f 1$ and $f 2$ are the chosen checkerboard frequencies, $17 \mathrm{~Hz}$ and $20 \mathrm{~Hz}$.

One of the most obvious distinctions between evoked responses to overt and covert attended stimuli is lateralization. The left and right visual fields are each processed by the visual cortex on the contralateral side of the brain, and the spatial distribution of evoked responses to stimuli tends to reflect this. In addition to this, the attentional modulation of SSVEPs has been found to be more pronounced on the side contralateral to the visual field of stimulation [8]. In light of this, a two-dimensional feature separating the two electrodes was also used in left/right classification:

$$
F 2(n)=\left[\log \left(\frac{X^{O 1}{ }_{n}(f 1)}{X^{O 1}{ }_{n}(f 2)}\right), \log \left(\frac{X^{O 2}{ }_{n}(f 1)}{X^{O 2}{ }_{n}(f 2)}\right)\right] .
$$

\section{E. Classification}

Linear discriminants were used as the classifier model for this study, providing a parametric approximation to Bayes' rule [17]. For a one-dimensional feature this corresponds to calculating a threshold in one dimension, thus feature F1 provided a simple solution for feedback. Optimisation of the linear discriminant model is achieved through direct calculation and is very efficient thus lending itself well to real-time applications.

Performance of the LDA classifier was assessed using 10 -fold cross validation [17]. This scheme randomly divides the available data into 10 approximately equal sized, mutually exclusive "folds". For a 10 -fold cross validation run, 10 classifiers are trained with a different fold used each time as the testing-set, while the other 9 folds are used for the training data. Cross validation estimates are generally pessimistically biased, as training is performed using a subsample of the available data. For each of the four task types, an equal number of left-attend and right-attend segments from all 8 trials were used in classification.

\section{RESUlts}

Due to excessive lateral eye movements resulting in an insufficient number of segments accepted $(<20$ per trial) after artifact rejection, the data of one subject were excluded from the analysis. The remaining 14 subjects demonstrated full compliance in the performance of the task, as reflected in the low number of overt eye movements and high percentage of correct reports of number of yellow stimuli appearing in the attended visual field for task types 2 and 4.
TABLE I

CLASSIFICATION ACCURACIES FOR FEATURE F1

\begin{tabular}{c|c|c|c|c}
\hline subject & Standard & Yellows & Feedback & $\begin{array}{c}\text { Feedback \& } \\
\text { Yellows }\end{array}$ \\
\hline 1 & 56.9 & 65.1 & 55.1 & 56.8 \\
2 & 53.0 & 51.5 & 56.7 & 57.6 \\
3 & 51.9 & 58.2 & 56.2 & 53.5 \\
4 & 57.3 & 58.5 & 50.2 & 55.3 \\
5 & 42.8 & 56.3 & 56.5 & 49.5 \\
6 & 47.7 & 55.1 & 56.1 & 55.9 \\
7 & 57.3 & 50.4 & 51.0 & 50.8 \\
8 & 60.4 & 56.5 & 47.9 & 57.4 \\
9 & 54.2 & 54.8 & 57.5 & 56.2 \\
10 & 55.4 & 52.2 & 59.2 & 52.4 \\
11 & 57.5 & 55.3 & 52.7 & 52.1 \\
12 & 48.5 & 51.8 & 60.3 & 45.9 \\
13 & 52.3 & 52.9 & 42.3 & 48.2 \\
14 & 64.9 & 57.0 & 61.2 & 52.1 \\
\hline average & 54.3 & 55.4 & 54.5 & 53.1 \\
\hline
\end{tabular}

Table 1 shows the classification accuracies for all 14 subjects using one-dimensional feature F1. The highest accuracy achieved by any subject using this feature extraction method was $65.1 \%$ for the "yellows" task type without feedback. Average accuracies for all task types are reduced considerably compared with overt attention results.

Table 2 shows the classification accuracies for all 14 subjects using two-dimensional feature F2. Average accuracies for all task types are marginally higher than those obtained using feature F1.

Subject reports following task completion indicated that the "yellows" task type was regarded as the "easiest". The "feedback" task type was regarded as "frustrating" due to variability of the feedback, and subjects tended to ignore auditory feedback while counting yellow stimuli in the "feedback and yellows" task type.

\section{DISCUSSION}

The results of this study show that, with no change to experimental setup or analysis strategy, disallowing overt eye movement in an SSVEP-based one-dimensional control scenario results in a reduction of left/right classification accuracy by about $20 \%$ on average. In light of this drop in performance, adjustments to the paradigm appear to be necessary.

The effects of extracting separate features from the two electrodes, exploiting lateralization effects, are demonstrated in this study. Though an improvement appears to result on average from using feature F2, this is insignificant in comparison to the overall reduction in performance using covert attention.

Rather than development of more sophisticated feature extraction methods, improvement of stimulus presentation used to elicit the SSVEP is most likely to improve performance. Whereas SSVEPs resulting from stimuli located in foveal vision are known to be of large amplitude and very robust $[6,13]$, similar stimuli located in peripheral 
TABLE II

CLASSIFICATION ACCURACIES FOR FEATURE F2

\begin{tabular}{c|c|c|c|c}
\hline subject & Standard & Yellows & Feedback & $\begin{array}{c}\text { Feedback \& } \\
\text { Yellows }\end{array}$ \\
\hline 1 & 59.6 & 65.6 & 54.1 & 58.2 \\
2 & 56.1 & 51.4 & 59.3 & 55.6 \\
3 & 55.1 & 57.4 & 60.5 & 55.4 \\
4 & 56.0 & 57.2 & 55.3 & 63.6 \\
5 & 53.2 & 59.9 & 54.4 & 49.1 \\
6 & 54.5 & 54.8 & 56.8 & 53.3 \\
7 & 63.5 & 57.0 & 50.4 & 55.0 \\
8 & 71.2 & 54.5 & 53.6 & 58.7 \\
9 & 66.0 & 58.1 & 61.8 & 59.7 \\
10 & 52.2 & 50.4 & 56.1 & 57.7 \\
11 & 54.4 & 57.4 & 58.3 & 50.7 \\
12 & 49.1 & 54.2 & 61.2 & 55.2 \\
13 & 52.9 & 59.5 & 49.3 & 53.7 \\
14 & 67.9 & 56.4 & 60.1 & 52.7 \\
\hline average & 58.0 & 56.7 & 56.5 & 55.6 \\
\hline
\end{tabular}

vision generate SSVEPs of much smaller amplitude. In order to elicit an SSVEP of an amplitude greater than the background EEG, stimulus presentation is required to be much more precise. For instance, checkerboard reversals in the setup used in this study were not locked to the refresh rate of the data projector used to display them. In the equivalent overt attention study, SSVEPs were robust to the resulting variations in luminance and frequency, and large enough so that relative amplitudes could still be used in classification. However, when covertly attended, imprecise stimuli can produce SSVEP amplitudes that are below the background EEG level, thus relative amplitude measures are confounded by random noise. In current covert attention studies currently under way, stimulus presentation is controlled in such a way that SSVEPs, and the relevant attentional modulations thereof, are much more pronounced.

Subject reports suggesting that continuous auditory feedback was not effective are undoubtedly due to the poor discriminating power of the feature controlling the feedback, as indicated by the classification accuracies for the "standard" task type. Thus no conclusions can yet be made on the potential for auditory feedback in a spatial selective attention task. This is currently under further investigation.

\section{CONCLUSION}

There is a considerable drop of $\sim 20 \%$ in performance of an SSVEP based BCI when eye movement is disallowed. This may be attributed to insufficient control and precision of stimulus presentation rather than shortcomings of the employed feature extraction method.

\section{ACKNOWLEDGMENT}

The authors would like to acknowledge Robert Burke of Media Lab Europe for the design of the signal processing framework facilitating stimulus presentation and online feedback.

\section{REFERENCES}

[1] J. R. Wolpaw, N. Birbaumer, D. J. McFarland, G. Pfurtscheller and T. M. Vaughan, "Brain-Computer Interfaces for Communication and Control." Clinical Neurophysiology, Vol. 113, Iss. 6, pp. 767-791. (2002).

[2] L. A. Farwell and E. Donchin, "Talking off the top of your head: toward a mental prosthesis utilizing event-related brain potentials," Electroencephalography and Clinical Neurophysiology, Vol. 70, Iss. 6, pp. 510-523. (1988).

[3] G. Pfurtscheller and C. Neuper, "Motor imagery and direct braincomputer communication," Proceedings of the IEEE, Vol. 89, Iss. 7, pp. 1123-1134. (2001).

[4] S. P. Kelly, D. Burke, P. deChazal and R. B. Reilly, "Parametric Models and spectral analysis for Classification in a BrainComputer Interface," Proc of the 14th International Conference on Digital Signal Processing, DSP2002, Vol. 1, pp 307-310, Santorini (2002)

[5] N. Birbaumer, A. Kubler, N. Ghanayim, T. Hinterberger, J. Perelmouter, J. Kaiser, I. Iversen, B. Kotchoubey, N. Neumann and H. Flor, "The thought translation device (TTD) for completely paralyzed patients," Neural Systems and Rehabilitation Engineering, IEEE Transactions on, Vol. 8, No. 2, pp. 190-193 (2000).

[6] D. Regan, Human Brain Electrophysiology: Evoked Potentials and Evoked Magnetic Fields in Science and Medicine. New York: Elsevier, 1989.

[7] C. Ming, G. Xiaorong, G. Shangkai, and X. Dingfeng, "Design and implementation of a brain-computer interface with high transfer rates," Biomedical Engineering, IEEE Transactions on, Vol. 49, No. 10, pp. 1181-1186. (2002)

[8] M. M. Muller, T. W. Picton, P. Valdes-Sosa, J. Riera, W. A. Teder-Salejarvi, S. A. Hillyard, "Effects of Spatial Attention on the Steady-State Visual Evoked Potential in the 20-30 Hz Range," Cognitive Brain Research, vol. 6, pp 249-261 (1998).

[9] R. B. Silberstein, J. Ciociari, A. Pipingas, "Steady-state visually evoked potential topography during the Wiscnosin Card sorting task," Electroencephalogr. Clin. Neurophys. 96 pp 24-35 (1995).

[10] S. T. Morgan, J. C. Hansen, S. A. Hillyard, "Selective attention to stimulus location modulates the steady state visual evoked potential," Proc. Natl. Acad. Sci. USA, 93, pp 4770-4774 (1996).

[11] G. Deco, O. Pollatos, J. Zihl, "The time course of selective attention: theory and experiments," Vision Research vol 42, pp 2925-2945 (2002).

[12] H. Helmholtz, Handbuch der physiologischen Optik, Leipzig:Voss (1867).

[13] E. Lalor, S. P. Kelly, C. Finucane, R. Burke, R. Smith, R. B. Reilly and G. McDarby, "Steady-state VEP-based Brain Computer Interface Control in an Immersive 3-D Gaming Environment," unpublished.

[14] American Electroencephalographic Society "Guidelines for Standard Electrode Position Nomenclature", J. Clin. Neurophysiol., 8(2): 200-202, (1991).

[15] Biopac Inc., Biopotential Amplifiers: http://www.biopac.com.

[16] National Instruments Inc., Data Acquisition Hardware: http://www.ni.com/dataacquisition.

[17] B.D. Ripley, Pattern Recognition and Neural Networks, Cambridge University Press. 1996. 